\title{
IMMUNOLOGY
}

\section{Toll-like receptors and antibody responses}

Arising from: C. Pasare \& R. Medzhitov Nature 438, 364-368 (2005).

Microbial components, such as lipopolysaccharides, augment immune responses by activating Toll-like receptors (TLRs). Some have interpreted this to mean that TLR signalling might not only help to initiate the adaptive immune response, but may also be required for it ${ }^{1}$. The expanded view is shared by Pasare and Medzhitov $^{2}$, who conclude from an analysis of mice deficient in MyD88 (a TLR-signalling adaptor protein) that the generation of $\mathrm{T}$ dependent antigen-specific antibody responses requires activation of TLRs in B cells. However, we show here that robust antibody responses can be elicited even in the absence of TLR signals. This appreciable TLR-independence of immune responses should be taken into account in the rational design of immunogenic and toleragenic vaccines.

In mice that lack both MyD88 and TRIF (another TLR-signalling adaptor protein), all known types of TLR signalling are absent ${ }^{3}$. We find that preimmune serum immunoglobulin concentrations are substantial in these mice, and that T-cell-dependent antibody responses

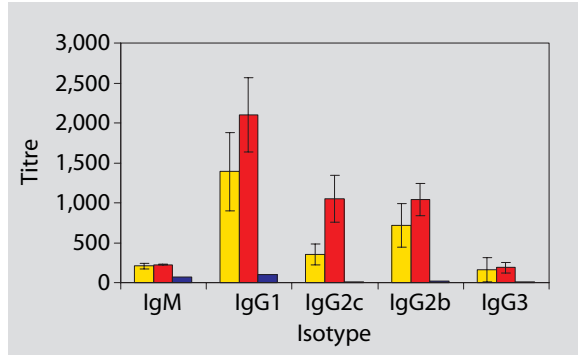

Figure 1 Antibody responses. T-cell-dependent antibody responses in wild-type $\mathrm{C} 57 \mathrm{BL} / 6$ mice (yellow) and in $\mathrm{MyD}^{-/-} / \mathrm{TRIF}^{\mathrm{Lps} 2 / \mathrm{Lps} 2}$ doubleknockout mice (red), which lack Toll-likereceptor activity. Young adult mice were given $100 \mu \mathrm{g}$ endotoxin-free trinitrophenolhaemocyanin conjugates in alum. At day 7 postimmunization, serum antibody responses of the antibody isotypes IgM, IgG1, IgG2c, IgG2b and IgG3 were quantified by using an enzyme-linked immunosorbent assay, with titres representing the dilution giving half-maximal optical density. Means and standard errors are shown for six mice per group. Sera from unimmunized C57BL/6 mice (blue) establish the background of the assay. to an antigen administered in alum are unimpaired (Fig. 1). It is notable that immunization with an antigen in alum, one of the few adjuvants approved for human use, promotes a TLR-independent antibody response. Further, we find that MyD88/TRIF double-deficient mice generate a robust antibody response to the T-independent antigen trinitrophenolFicoll (results not shown).

In the final analysis, we believe that Pasare and Medzhitov have simply demonstrated that TLR-dependent B-cell responses depend upon TLR signalling. But TLR signalling is clearly not essential for a response to typical vaccination. D. Nemazee, A. Gavin, K. Hoebe, B. Beutler Department of Immunology, The Scripps Research Institute, La Jolla, California 92037, USA e-mail: nemazee@scripps.edu 1. Medzhitov, R. \& Janeway, C. A. Jr Science $\mathbf{2 9 6 , 2 9 8 - 3 0 0}$
(2002).
2. Pasare, C. \& Medzhitov, R. Nature 438, 364-368 (2005).
3. Hoebe, K. et al. Nature 424, 743-748 (2003).

doi:10.1038/nature04875

\section{IMMUNOLOGY}

\section{Pasare \& Medzhitov reply}

\section{Replying to: D. Nemazee, A. Gavin, K. Hoebe \& B. Beutler Nature 441, doi:10.1038/nature04875 (2006)}

Using a range of different experimental approaches (and not just analyses of MyD88deficient mice), we showed that, contrary to the prevailing view, a T-helper response is not sufficient for the generation of optimal Tdependent antibody responses, and that Tolllike-receptor (TLR) signalling in B cells is also required $^{1}$. Similar results have since been obtained in human B cells ${ }^{2}$. Nemazee et al. ${ }^{3}$ now question our conclusions ${ }^{1}$.

We showed that the requirement for TLR signalling in antibody responses depends on the antibody isotype ${ }^{1}$ : the IgM and IgG1 isotypes are largely, but not completely, TLRdependent; IgG2 isotypes are entirely TLR-dependent; and IgE and IgA responses are TLR-independent. But in any event, our main discovery was that TLR signalling has to happen in B cells in order for the optimum Tcell-dependent antibody response to be generated. It was not, as claimed by Nemazee et al. ${ }^{3}$, simply that TLR-dependent B-cell responses depend on TLR signalling.

Figure 1 of Nemazee et al. ${ }^{3}$ shows responses obtained under suboptimal conditions, without essential comparisons and controls ${ }^{1}$ : for example, their antibody responses would seem negligible if compared with the response induced in the presence of TLR ligands. The results do not therefore present a meaningful counterargument to our conclusions.

Nemazee et al. claim that TLR signalling is not essential for responses to "typical vaccination", which very much depends on what is meant by 'typical'. Most successful vaccines, such as attenuated pathogens, certainly do trigger TLR signalling, which is one of the reasons why they are successful. The subunit vaccines, on the other hand, generally do not work; the few that do, do so because they either activate TLRs or some TLR-equivalent component of the innate immune system.

\section{Pasare, R. Medzhitov}

Howard Hughes Medical Institute and

Section of Immunology, Yale University

School of Medicine, New Haven, Connecticut

06510, USA

e-mail: medzhitov@yale.edu

1. Pasare, C. \& Medzhitov, R. Nature 438, 364-368 (2005).

2. Ruprecht, C. R. \& Lanzavecchia, A. Eur. J. Immunol. 36, 810-816 (2006).

3. Nemazee, D., Gavin, A., Hoebe, K. \& Beutler, B. Nature 441, doi:10.1038/nature04875 (2006).

doi:10.1038/nature04876 